\title{
Avaliação da resistência residual de lajes alveolares em concreto armado em uma edificação industrial após incêndio
}

\author{
Residual strength evaluation of hollow core \\ slabs of reinforced concrete of an industrial \\ building after fire
}

Hinoel Zamis Ehrenbring ${ }^{1}$ Vinicius Ortolan ${ }^{2}$ Fabricio Bolina ${ }^{3}$ Fernanda Pacheco ${ }^{4}$ Augusto Masiero Gil ${ }^{5}$ Bernardo Fonseca Tutikian ${ }^{6}$

\footnotetext{
${ }^{1}$ Engenheiro Civil, mestrando em Engenharia Civil (Programa de Pós-Graduação em Engenharia Civil - Universidade do Vale do Rio dos Sinos (UNISINOS), São Leopoldo, RS.

e-mail: hinoelzamis@hotmail.com

${ }^{2}$ Engenheiro Civil, Mestre em Engenharia Civil (Programa de Pós-Graduação em Engenharia Civil - Universidade do Vale do Rio dos Sinos (UNISINOS), São Leopoldo, RS.

e-mail: vortolan88@gmail.com

${ }^{3}$ Engenheiro Civil, Mestre em Engenharia Civil (Programa de Pós-Graduação em Engenharia Civil - Universidade do Vale do Rio dos Sinos (UNISINOS), São Leopoldo, RS.

e-mail: fabriciobolina@gmail.com

${ }^{4}$ Engenheira Civil, Mestre em Engenharia Civil (Programa de Pós-Graduação em Engenharia Civil - Universidade do Vale do Rio dos Sinos (UNISINOS), São Leopoldo, RS.

e-mail: fernandapacheco.fe@gmail.com

${ }^{5}$ Engenheiro Civil, mestrando em Engenharia Civil (Programa de Pós-Graduação em Engenharia Civil - Universidade do Vale do Rio dos Sinos (UNISINOS), São Leopoldo, RS.

e-mail: augusto.masiero@hotmail.com

${ }^{6}$ Professor Dr. Programa de Pós Graduação em Engenharia civil (itt Performance / Unisinos)

e-mail: bftutikian@unisinos.br
}

\section{RESUMO}

O concreto, durante exposição a elevadas temperaturas, caracteriza-se pela baixa difusividade térmica e incombustibilidade, resultando em desempenho satisfatório frente ao fogo. Todavia, constatam-se transformações químicas e físicas em seus componentes. A estabilidade do composto relaciona-se com a microestrutura, portanto consegue-se verificar a degradação do material através de técnicas avançadas de análise microestrutural. Neste contexto, o uso de ensaios no material, como a difratometria e fluorescência de raios X, mostrase atraente. Neste artigo, é descrita a inspeção de laje alveolar pré-fabricada de uma edificação industrial, a qual sofreu exposição às altas temperaturas provindas de um incêndio no subsolo da edificação. Avaliou-se o elemento estrutural através de ensaios de caracterização química, em diferentes espessuras da laje de concreto, estimando a temperatura alcançada em cada camada e, consequentemente, a perda de resistência do elemento estrutural. A partir dos resultados, constatou-se a temperatura de, aproximadamente, $700{ }^{\circ} \mathrm{C}$ na superfície da laje e menos de $100{ }^{\circ} \mathrm{C}$ nas camadas mais profundas. Estimou-se a redução na resistência à compressão do concreto na ordem de $25 \%$ na camada de $20 \mathrm{~mm}$ e redução praticamente desprezável na região dos fios protendidos, atestando a segurança estrutural da estrutura após o sinistro.

Palavras-chave: Concreto. Altas temperaturas. Resistência residual. Difração por raios-x. Fluorescência de raios $\mathrm{x}$.

\section{ABSTRACT}

Concrete, during exposure to elevated temperatures, is characterized by low thermal diffusivity and incombustibility, resulting a satisfactory performance against fire. However, chemical and physical changes occur in its components. The stability of the compound relates to its microstructure, so it is possible to verify the material degradation using advanced techniques of microstructural analyses. In this context, the use of mate- 
rials tests, such as diffraction and fluorescence $\mathrm{X}$-ray, proves attractive. In this paper, it is described the prefabricated cellular slab inspection, an industrial building, which has undergone exposure to high temperatures emanating from a fire in the building ground. It was evaluated the structural element by chemical characterization tests with different thickness of the concrete slab, estimating the temperature reached in each layer and, consequently, the resistance loss of the structural element. From the results, it was found the temperature of approximately $700{ }^{\circ} \mathrm{C}$ in the slab surface and less than $100{ }^{\circ} \mathrm{C}$ in the deeper layers. It was estimated the reduction in strength of concrete in the order of $25 \%$ in $20 \mathrm{~mm}$ layer and negligible reduction in the region of prestressed wire, attesting to the structural safety of the structure after the accident.

Keywords: Concrete. High temperatures. Residual resistance. X- ray diffraction. Fluorescence x-ray.

\section{INTRODUÇÃO}

O concreto caracteriza-se por ser um material constituído por diferentes fases, sendo elas os agregados, a matriz cimentícia e a zona de transição entre estes componentes. [1]. Devido a sua estrutura complexa e heterogênea, tem-se dificuldade em estimar suas reações e tipos de deterioração quando submetido a diferentes condições, tal como em altas temperaturas. Porém, as reações podem ser identificadas através de uma análise microestrutural dos produtos resultantes das transformações dos compostos hidratados, sendo possível estimar a integridade dos elementos assim concebidos. [2].

Para Morales, Campos e Faganello [3], a heterogeneidade da mistura acarreta na diferença de intensidade das reações já conhecidas destes compostos no momento em que são submetidas a elevados patamares de temperatura. Os autores reforçam a importância deste tipo de estudo, já que na ocorrência de incêndio o desempenho dos elementos pode tornar-se insuficiente para as solicitações às quais foram projetados. Sabe-se que o concreto apresenta menor condutividade térmica quando comparado ao aço, ou seja, apresentando um adequado desempenho térmico. Além disso, as matrizes cimentícias, em geral, apresentam incombustibilidade e baixa difusividade térmica, não emitindo fumaça e proporcionando um isolamento térmico adequado. [4].

Quando exposto a altas temperaturas, o concreto é capaz de manter parte da sua resistência inicial sem perda de estabilidade estrutural. [5]. Bolina et. al. [6] apresentam um estudo relacionando a resistência ao fogo de paredes maciças de concreto, demonstrando a eficiência deste sistema por períodos de até 120 minutos. No entanto, diversos fatores, como a temperatura máxima atingida, o tempo de exposição, a composição da mistura, o tipo de estrutura e a velocidade de resfriamento podem ocasionar a redução da resistência ao fogo. [3].

As consequências da exposição de matrizes cimentícias a altas temperaturas são representadas através das modificações das propriedades físicas e químicas do material. A composição química é afetada com a transformação de minerais à medida que se eleva a temperatura e ultrapassa o patamar de estabilidade térmica dos elementos. Já as alterações físicas são promovidas pelo aparecimento de fissuras e a ocorrência do desplacamento do material. [7, 8].

O fenômeno de desplacamento do concreto manifesta-se através da perda das camadas superficiais, podendo ocorrer de forma instantânea e violenta ou gradual e progressiva. Em meio a esta instabilidade comportamental, sabe-se que o desplacamento origina-se próximo à superfície do elemento de concreto, quando o mesmo é sujeito a gradientes térmicos elevados. $[9,10,11]$. O desplacamento é um fenômeno presente nos compostos cimentícios e mais suscetível em concretos que possuem baixa relação água/cimento, por apresentarem menor quantidade de poros, possibilitando a interconectividade entre os vazios. [12, 13, 14]. Durante o aquecimento, a água livre entra em expansão, decorrente do processo de evaporação, aumentando a pressão interna dos poros, que, quando superam a resistência à tração do material, ocasionam o seu desplacamento. [15].

Ainda que o concreto apresente um comportamento adequado frente ao fogo, Fib [16] e Lim e Mondal [17] enfatizam que o aumento da temperatura dos elementos estruturais causa alterações em sua micro e macroestrutura. A sensibilidade destas microestruturas pode ser aferida por meio de procedimentos experimentais que identificam as alterações físicas, químicas e mineralógicas da matriz cimentícia, como é o caso dos ensaios de difratometria e fluorescência por raios X. [18]. Tomando como parâmetro a execução destes ensaios, existe a possibilidade de estimar-se a temperatura em que o material foi submetido.

De acordo com Minami et al. [19], a evaporação da água livre na pasta de cimento ocorre aos $100^{\circ} \mathrm{C}$. A perda da estabilidade da pasta se inicia entre $100{ }^{\circ} \mathrm{C}$ e $200{ }^{\circ} \mathrm{C}$. Acima dos $200{ }^{\circ} \mathrm{C}$ iniciam-se as reações físico-química e a água evaporada reduz as forças de Van der Walls entre as camadas de C-S-H. [20]. Entre os $400{ }^{\circ} \mathrm{C}$ e $600{ }^{\circ} \mathrm{C}$ ocorrem diversas transformações na pasta de cimento: dessecação dos poros, seguida da 
decomposição dos produtos de hidratação e destruição do gel de C-S-H, reação endotérmica da desidratação do hidróxido de cálcio $\left(\mathrm{Ca}(\mathrm{OH})_{2}\right)$ e liberação o óxido de cálcio $(\mathrm{CaO})$. [21].

Em termos da análise das alterações causadas pela ação do fogo, a NBR 15200 [22], de modo similar ao Eurocode 2 [23], apresenta uma estimativa da redução da resistência à compressão do concreto endurecido em função do acréscimo de temperatura, considerando um coeficiente redutor $\mathrm{K}_{\mathrm{c}}, \Theta$, apresentado na Tabela 1. Tem-se, desta forma, a estimativa do grau de dano mecânico causado e a resistência residual do concreto, relacionando-a com as temperaturas que o elemento estrutural foi exposto.

Portanto, o presente trabalho objetiva avaliar a resistência residual de um elemento estrutural de concreto armado após a ocorrência de um incêndio, de modo que se possa identificar se são necessárias intervenções para reabilitação da estrutura. Esta dedução apoiou-se em análises visuais e em ensaios de difratometria de raios $\mathrm{X}$ e de fluorescência de raios $\mathrm{X}$, seguido da estimativa da temperatura máxima que a estrutura foi incidida para correlacionar com da perda de resistência do concreto e das armaduras, embasados nas informações da ABNT NBR 15200 [22] e Eurocode 2 [23].

\section{MATERIAIS E MÉTODOS}

A edificação industrial avaliada sofreu um incêndio em seu subsolo, local usado para a galvanoplastia e eletrólise de materiais com o uso de ligas metálicas. A edificação foi executada em estrutura de concreto armado pré-fabricada, utilizando laje alveolar com espessura de $26,5 \mathrm{~cm}$, fios protendidos de diâmetro de $12,7 \mathrm{~mm}$ e cobrimento de concreto de $2,5 \mathrm{~cm}$. A resistência à compressão característica do concreto da laje é de $30 \mathrm{MPa}$. A seção transversal da laje avaliada é representada no corte esquemático da Figura 1.

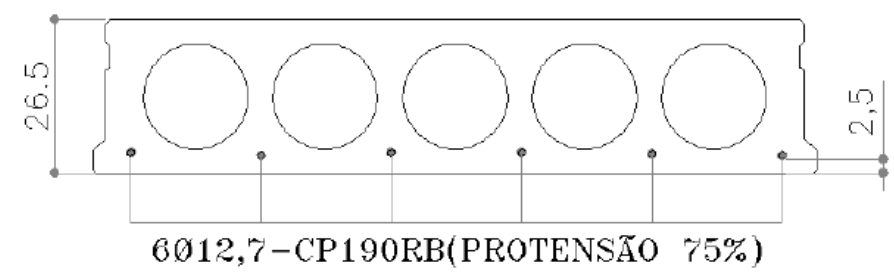

Figura 1: Detalhamento estrutural da laje (medidas em $\mathrm{cm}$ )

A incidência das chamas ocorreu na parte inferior da laje do pavimento sinistrado, tendo como região mais afetada a sobre o tanque de cromo. A Figura 2 ilustra a área após o incêndio.
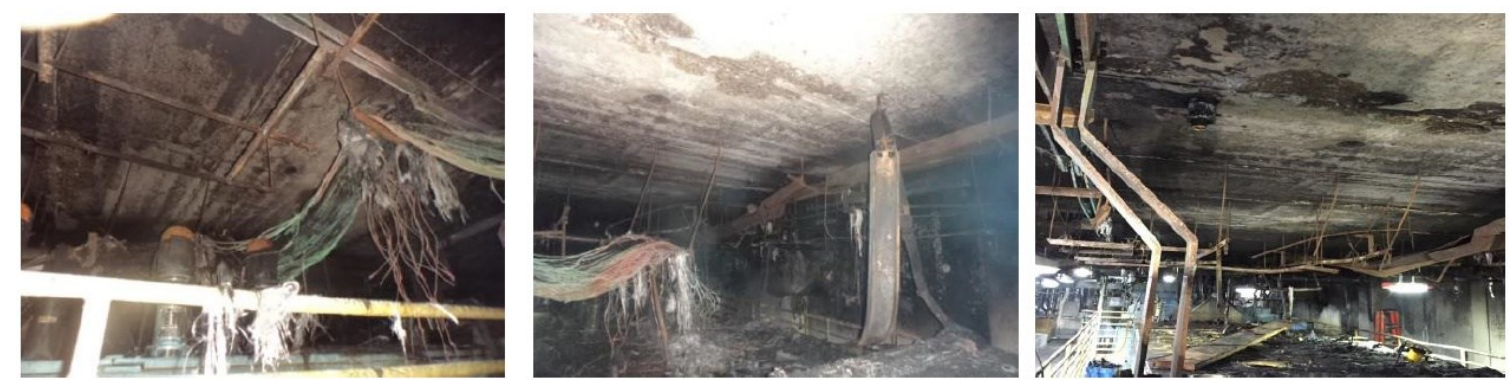

Figura 2: Estado geral da laje sinistrada do subsolo da edificação

A partir de uma aferição visual definiu-se como o foco do incêndio o local próximo ao tanque de cromo, de área crítica aproximada de $20 \mathrm{~m}^{2}$. Desde o princípio das chamas, até o início do seu combate, transcorreu-se 30 minutos. Uma das manifestações patológicas observadas na área das lajes foi o desplacamento do concreto, conforme ilustrado na Figura 3. 


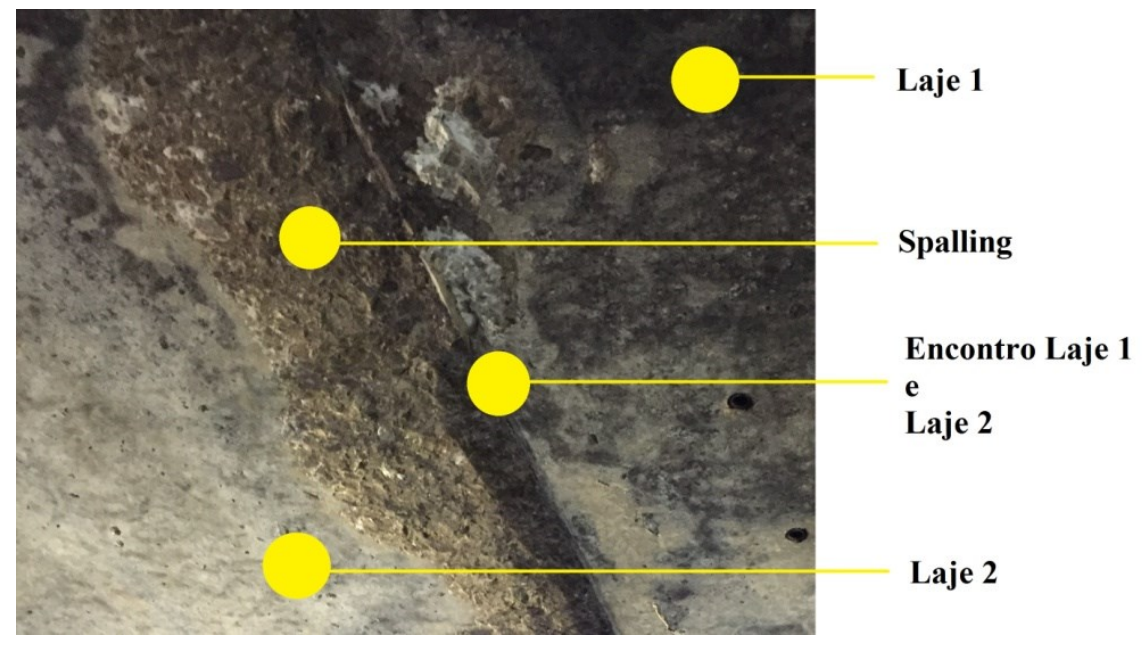

Figura 3: Desplacamento do concreto na superfície inferior da laje exposta a ação do fogo

Com base nas informações obtidas, consideraram-se as lajes como os elementos estruturais mais afetados, por apresentarem maior tempo de permanência sob a ação do fogo. Em virtude deste fato, optou-se pela avaliação do concreto das lajes alveolares. A fim de deduzir o estado de integridade destes elementos, foram realizadas extrações de $60 \mathrm{~g}$ de massa em diversos pontos e em distintas profundidades da laje, até uma espessura de $40 \mathrm{~mm}$. Os exemplares foram extraídos a partir da face inferior da laje em um trecho com $50 \%$ da área total do elemento sinistrado, nas profundidades de 10, 20, 30 e $40 \mathrm{~mm}$, conforme apresentado na Figura 4.
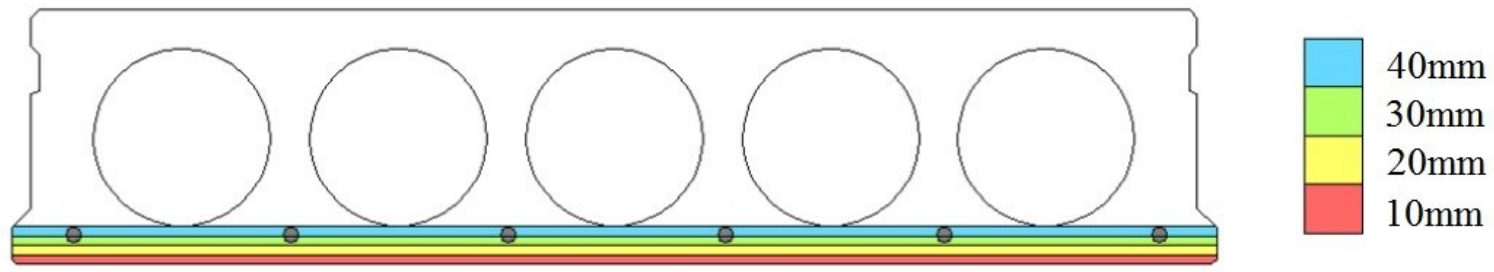

Figura 4: Profundidades analisadas

As amostras consistiram de um material pulverulento. Os pontos de extração foram definidos com o auxílio de um detector digital magnético, de modo que o processo de coleta não danificasse a armadura constituinte da laje. A Figura 5 detalha o trabalho das equipes na coleta dos exemplares. Com o material coletado foi realizada a análise laboratorial do concreto e das transformações físico-químicas deste material.
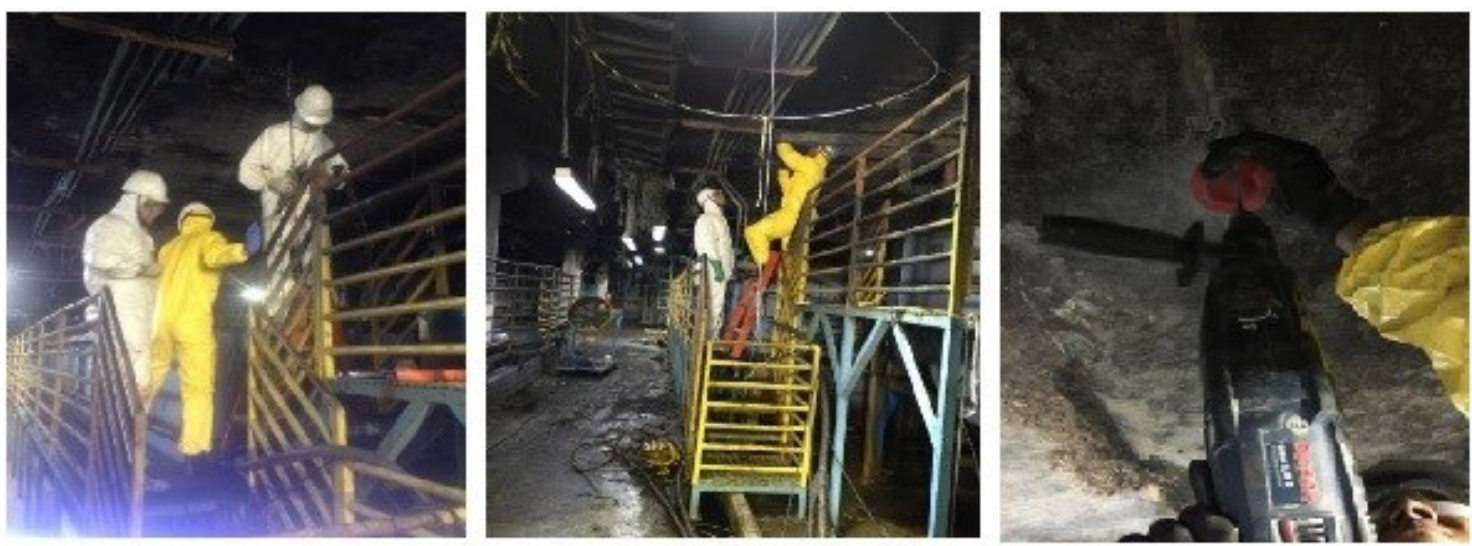

Figura 5: Detalhe na extração dos exemplares da amostra. 
O material pulverulento extraído foi separado em recipientes selados e identificados, sendo transportados até o laboratório para análise. Após o processo de extração e transporte do material, o mesmo sofreu uma preparação preliminar antes de ser submetido aos ensaios de fluorescência e difratometria de raios X. A preparação dos exemplares foi feita através de sua moagem, em almofariz de Ágata, conforme Figura 6, garantindo a finura adequada para os ensaios.

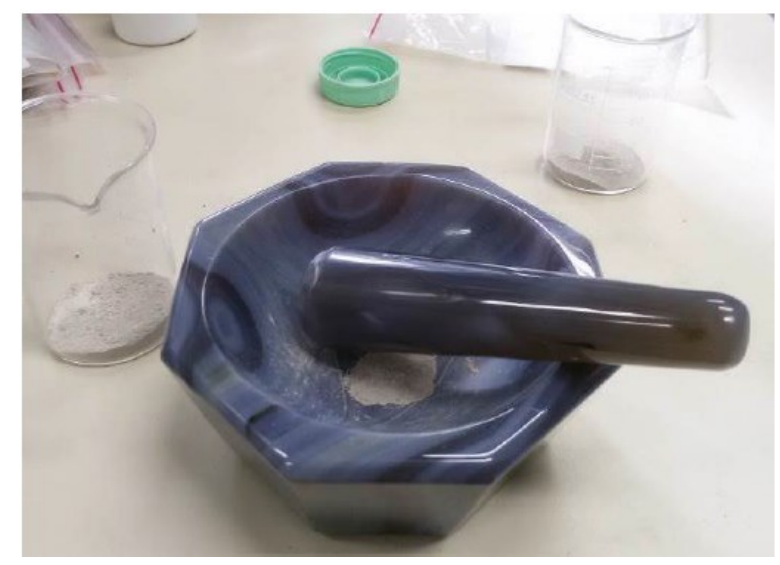

Figura 6: Moagem do material pulverulento com Gral de Ágata

$\mathrm{O}$ ensaio de fluorescência de raios $\mathrm{X}$ foi realizado para a determinação e identificação dos minerais constituintes, visando caracterizar a microestrutura cristalina. O equipamento utilizado para esta análise foi o espectrômetro de fluorescência de raios X, marca EDX 720HS. A quantidade necessária de amostras para a realização dos ensaios em cada profundidade é de $5 \mathrm{~g}$.

De modo análogo, e complementando a caracterização dos testemunhos, o ensaio de difratometria de raios $\mathrm{X}$ foi empregado para obter informações sobre a estrutura atômica e molecular do material coletado, viabilizando a identificação da composição química do concreto de cada camada e de suas fases cristalinas. Empregou-se para este ensaio o difratômetro de raios X, marca Siemens, modelo D5000. Para a realização deste ensaio é necessário, no mínimo, $10 \mathrm{~g}$ de material, para cada amostra. Para interpretação dos dados obtidos foi utilizado o programa PANalytical X'Pert HighScore.

Finalizados os ensaios de análise química e mineralógica das amostras, identificam-se os prováveis níveis de temperaturas alcançados e determina-se a provável resistência residual através da Tabela 1, oriunda da ABNT NBR 15200 [22].

Tabela 1: Valor de resistência à compressão em função da temperatura na estrutura de concreto

\begin{tabular}{c|c}
$\begin{array}{c}\text { TEMPERATURA } \\
\text { DO CONCRETO } \\
\left({ }^{\circ} \mathbf{C}\right)\end{array}$ & $\mathbf{K}_{\mathbf{c}}, \boldsymbol{\Theta}=\mathbf{f}_{\mathbf{c}}, \boldsymbol{\Theta} / \mathbf{f c k}$ \\
\hline 1 & 2 \\
\hline 20 & 1 \\
\hline 100 & 1 \\
\hline 200 & 0,95 \\
\hline 300 & 0,85 \\
\hline 400 & 0,75 \\
\hline 500 & 0,6 \\
\hline 600 & 0,45 \\
\hline 700 & 0,3 \\
\hline 800 & 0,15 \\
\hline 900 & 0,08 \\
\hline 1000 & 0,04 \\
\hline 1100 & 0,01 \\
\hline 1200 & 0 \\
\hline
\end{tabular}

Conforme expressa a tabela acima, os valores expressos na coluna direita correspondem ao coeficiente $\mathrm{K}_{\mathrm{c}}, \Theta$. Tal coeficiente é um fator de correção, estimando de maneira empírica, a perda de resistência do concreto em função das temperaturas em que é exposto. 


\section{RESULTADOS}

A partir do ensaio de fluorescência de raios-X identificaram-se os elementos químicos presentes em cada uma das amostras, para cada profundidade analisada, conforme expressa a Tabela 2.

Tabela 2: Elementos químicos identificados nas amostras extraídas

\begin{tabular}{c|c|c|c}
\multirow{2}{*}{$\begin{array}{c}\text { PROFUNDIDADE } \\
(\mathbf{m m})\end{array}$} & \multicolumn{3}{|c}{ ELEMENTOS (\%) } \\
\cline { 2 - 4 } & $\mathbf{( > \mathbf { 5 0 } )}$ & $\mathbf{( 5 < \mathbf { x } < \mathbf { 5 0 } )}$ & $\mathbf{( < \mathbf { 5 } )}$ \\
\hline 40 & Não identificados & $\mathrm{Si}, \mathrm{Ca}, \mathrm{Fe}, \mathrm{Al}$ & $\mathrm{K}, \mathrm{Mg}, \mathrm{Ti}, \mathrm{S}, \mathrm{Mn}, \mathrm{Sr}, \mathrm{Zr}, \mathrm{Rb}$ \\
\hline 30 & Não identificados & $\mathrm{Si}, \mathrm{Ca}, \mathrm{Fe}, \mathrm{Al}$ & $\mathrm{K}, \mathrm{Mg}, \mathrm{Ti}, \mathrm{S}, \mathrm{Mn}, \mathrm{Sr}, \mathrm{Rb}$ \\
\hline 20 & Não identificados & $\mathrm{Si}, \mathrm{Ca}, \mathrm{Fe}, \mathrm{Al}$ & $\mathrm{K}, \mathrm{Mg}, \mathrm{Ti}, \mathrm{S}, \mathrm{Mn}, \mathrm{Sr}, \mathrm{Zr}, \mathrm{Rb}$ \\
\hline 10 & Não identificados & $\mathrm{Si}, \mathrm{Ca}, \mathrm{Fe}, \mathrm{Al}$ & $\mathrm{K}, \mathrm{Mg}, \mathrm{Ti}, \mathrm{S}, \mathrm{Ba}, \mathrm{Mn}, \mathrm{Sr}$ \\
\hline
\end{tabular}

A fim de complementar os resultados obtidos no ensaio de fluorescência de raios X, adotou-se também como parâmetro avaliativo o ensaio de difratometria de raios X. A Figura 7, 8, 9 e 10 são referentes a este ensaio, cujos testemunhos também foram extraídos das profundidades de 10, 20, 30 e $40 \mathrm{~mm}$, respectivamente.
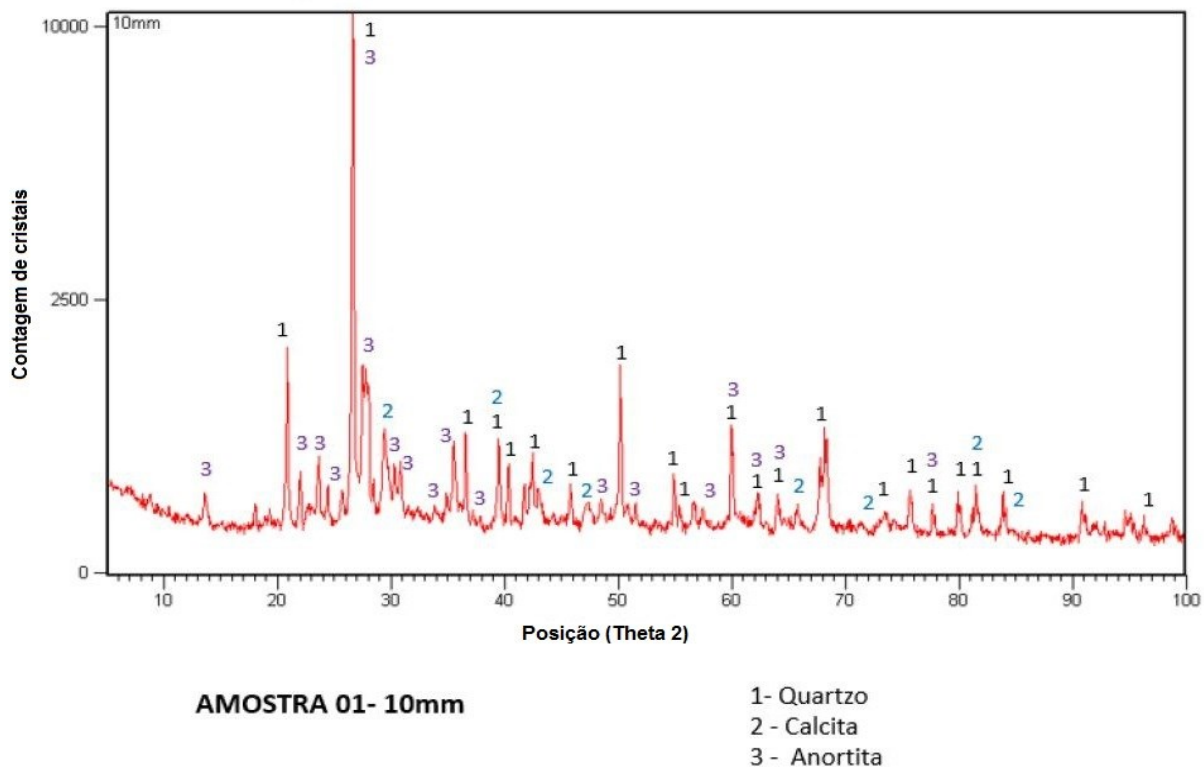

Figura 7: Difração de Raios-X - Amostra com profundidade de $10 \mathrm{~mm}$

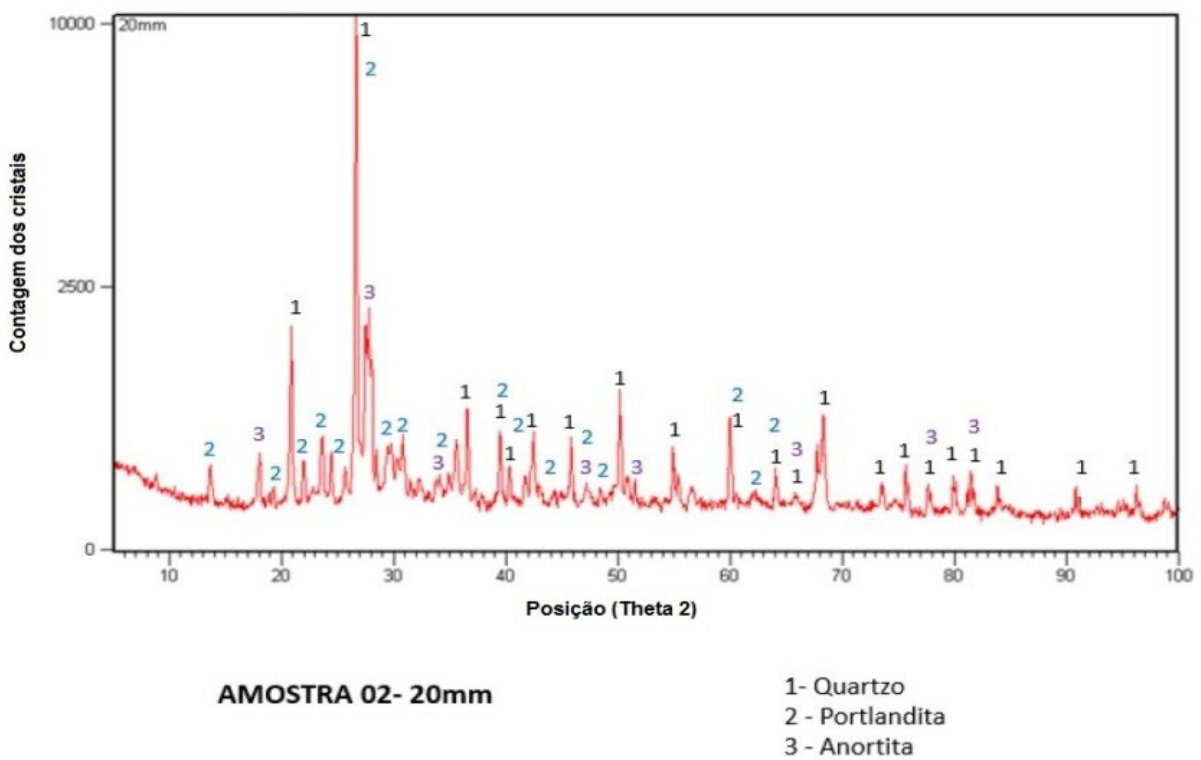

Figura 8: Difração de Raios-X - Amostra com profundidade de $20 \mathrm{~mm}$ 


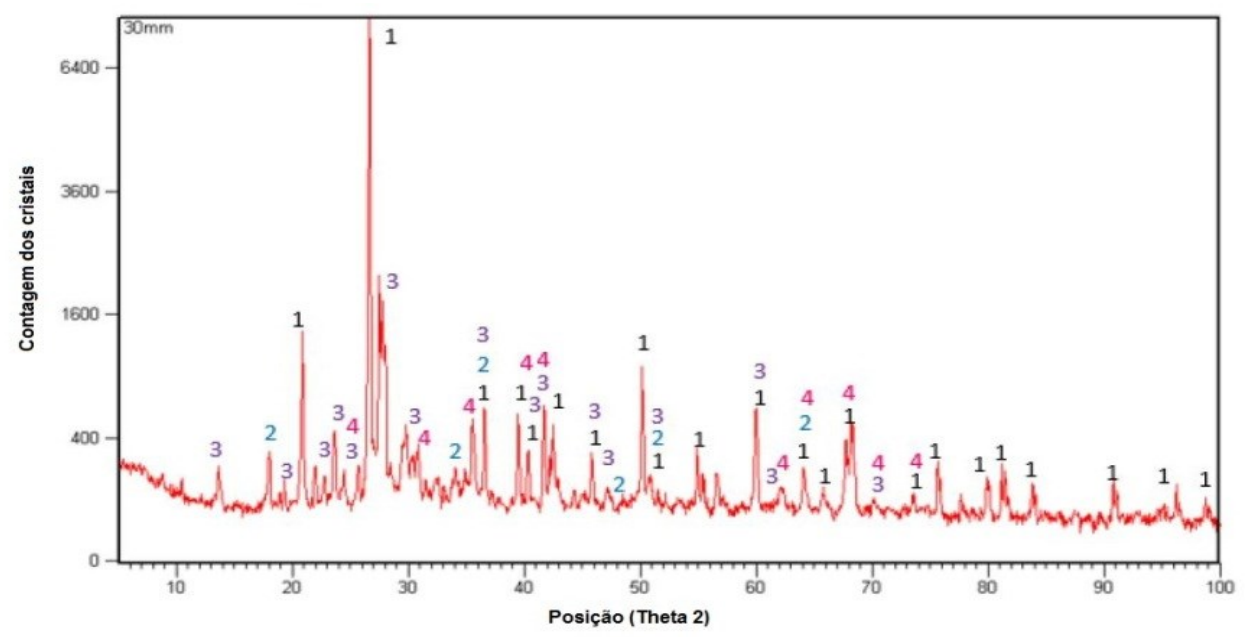

AMOSTRA 03- $30 \mathrm{~mm}$

1- Quartzo

2 - Portlandita

3 - Anortita

4 - Diopsida

Figura 9: Difração de Raios-X - Amostra com profundidade de $30 \mathrm{~mm}$

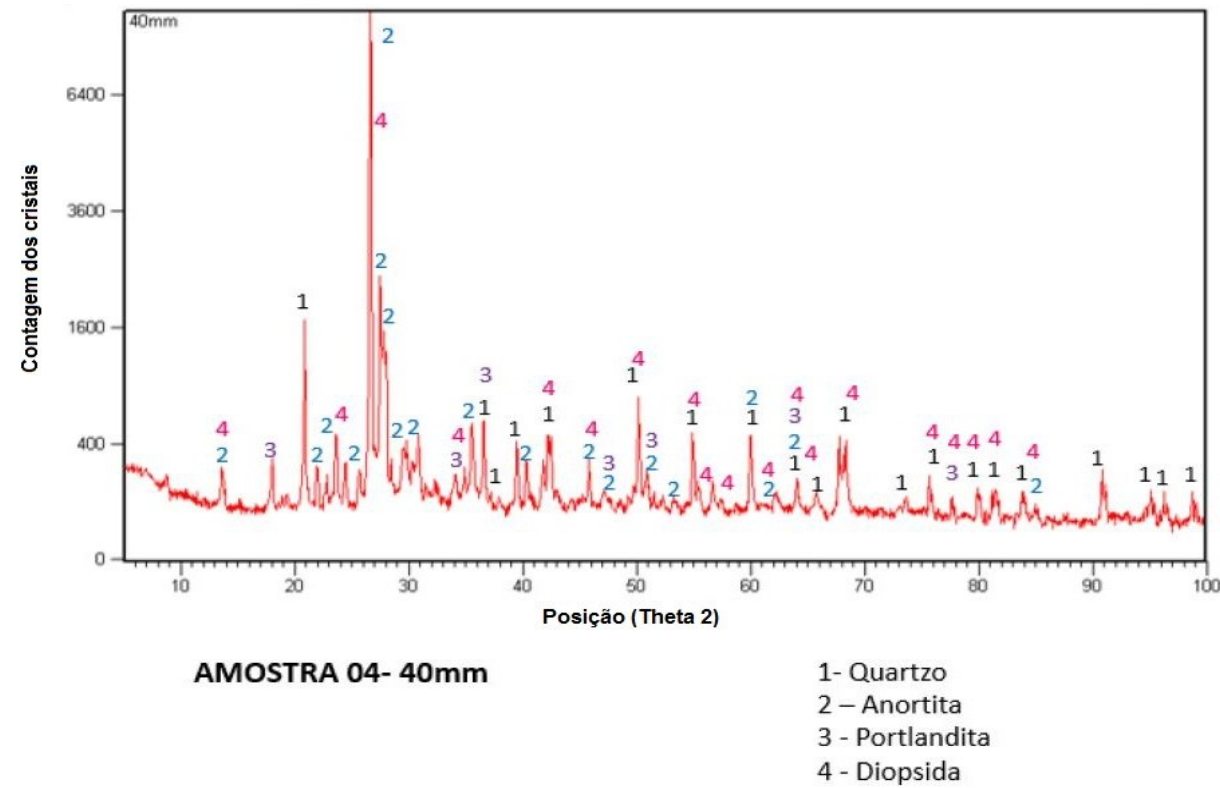

Figura 10: Difração de Raios- $X$ - Amostra com profundidade de $40 \mathrm{~mm}$

A Tabela 3 ilustra os valores das temperaturas identificadas através dos ensaios de fluorescência e difratometria de raios $\mathrm{X}$.

Tabela 3: Distribuição de temperaturas na seção da laje sinistrada.

\begin{tabular}{c|c|c|c|c}
$\begin{array}{c}\text { PROFUNDIDADE } \\
(\mathbf{m m})\end{array}$ & $\begin{array}{c}\text { TEMPERATURA } \\
\text { PROVÁVEL } \\
\left({ }^{\mathbf{0}} \mathbf{C}\right)\end{array}$ & $\begin{array}{c}\text { PERDA DE RESISTÊN- } \\
\text { CIA PROVÁVEL DO } \\
\text { CONCRETO } \\
\mathbf{( \% )}\end{array}$ & $\begin{array}{c}\text { TEMPERATURA } \\
\text { PROVÁVEL AR- } \\
\text { MADURAS ( } \mathbf{C})\end{array}$ & $\begin{array}{c}\text { PERDA DE RESIS- } \\
\text { TENCIA PROVÁVEL } \\
\text { DAS ARMADURAS (\%) }\end{array}$ \\
\hline 10 & $<700$ & 70 & Sem armaduras & Sem armaduras \\
\hline 20 & $<400$ & 25 & $400^{\circ} \mathrm{C}$ & 40 \\
\hline 30 & $<100$ & Desprezável & - & - \\
\hline 40 & $<100$ & Desprezável & - & - \\
\hline
\end{tabular}




\section{DISCUSSÃO}

O silício ( $\mathrm{Si}$ ), presente em quantidades maiores do que $5 \%$ e menores do que $50 \%$, possivelmente origina-se dos agregados, conforme Handoo e Agarwal [24]. Não foram encontrados teores de carbono em quantidades superiores a $5 \%$ em qualquer camada, remetendo à indicação de que as temperaturas das camadas mais internas do elemento não atingiram temperaturas superiores $800{ }^{\circ} \mathrm{C}$, assim como evidenciaram Lima [25] e Sousa [26]. Nota-se, ademais, que, pela marcante quantidade de silício ( $\mathrm{Si})$, cálcio $(\mathrm{Ca})$, ferro $(\mathrm{Fe})$ e alumínio (Al) têm-se indícios da presença dos produtos de hidratação, indicando, através das quantidades observadas e conforme afirmações feitas por Arioz [27] e Kim, Yun e Park [28], altas concentrações de silicato de cálcio hidratado (C-S-H), o que é positivo para a estrutura, uma vez que este elemento é relacionado com a resistência do material.

Tomando como base as Figuras 7, 8, 9 e 10 pode-se identificar os indícios das temperaturas reais em cada amostra, devido à extinção de algumas composições mineralógicas.

O material contido na camada de $10 \mathrm{~mm}$ não apresentou índices químicos contendo portlandita $\left[\mathrm{Ca}(\mathrm{OH})_{2}\right]$ na sua composição, remetendo à indicação que, nesta espessura, as temperaturas foram superiores a $420^{\circ} \mathrm{C}$, atingindo, provavelmente $700{ }^{\circ} \mathrm{C}$, uma vez que este é ponto de início do processo de transformação do hidróxido de cálcio $(\mathrm{CH})$ e de extinção completa da portlandita. [18]. A inexistência de entringita nesta camada confirma esta presunção, podendo ser um indício de que a temperatura superou $350{ }^{\circ} \mathrm{C}$, como comprovam Annerel e Taerwe [29]. Esta interpretação foi reforçada pela presença de calcita $\left(\mathrm{CaCO}_{3}\right)$.

Nas espessuras de 20, 30 e $40 \mathrm{~mm}$ notou-se a presença do hidróxido de cálcio, incrementado em termos de identificação dos picos, com o aumento da profundidade. Não se observou carbonato de cálcio ou óxido de cálcio nas camadas superiores a $20 \mathrm{~mm}$. Estes elementos são oriundos da desintegração do C-S-H, que ocorre em temperaturas superiores a $700{ }^{\circ} \mathrm{C}$. [17]. Esta interpretação remete que, nestas camadas, as temperaturas não chegaram neste limite. $\mathrm{O}$ hidróxido de cálcio observado indica que as temperaturas não foram superiores a $400^{\circ} \mathrm{C}$ nestas camadas, assim como visto por Annerel e Taerwe [29].

Nas camadas de $30 \mathrm{~mm}$ e $40 \mathrm{~mm}$ ainda se identificou os compostos da pasta hidratada de cimento. Este fato indica que o calor não promoveu profundas alterações químicas na pasta, e consequentemente mecânicas, sendo possível projetar que a temperatura atingida foi inferior a $100{ }^{\circ} \mathrm{C}$, tendo como base Sousa [26] e Kim, Yun e Park [28]. O próprio grau do desplacamento do concreto observado in loco se limitou a uma espessura de $20 \mathrm{~mm}$, confirmando a análise.

Analisando os resultados laboratoriais mencionados na Tabela 3 pressupõe-se que, nas camadas mais profundas do que $20 \mathrm{~mm}$ da laje, as temperaturas não ultrapassaram $400{ }^{\circ} \mathrm{C}$. Segundo a ABNT NBR 15200 [22], nesta faixa, o concreto tem um provável coeficiente de redução de resistência à compressão de 0,75 , com perda de $25 \%$ da sua resistência inicial em temperatura ambiente. Este coeficiente é tratado como uma estimativa razoável da projeção das perdas de resistências, visto que este parâmetro depende, além da natureza dos materiais constitutivos do concreto, da idade do elemento estrutural, da forma de exposição às chamas, do grau de restrição à deformação linear, geometria, entre outros.

Bolina [14] aponta que, nas estruturas de concreto armado, as temperaturas das armaduras tendem a ser ligeiramente superiores as do concreto, dada a sua maior condutividade térmica. As fissuras pré-existentes ou desenvolvidas no concreto durante o seu aquecimento auxiliam numa condução de calor direta. Cabe destacar que a redução de resistência estimada se refere àquela verificada no ato do aquecimento. Após o resfriamento, $\mathrm{o}$ aço tende a recuperar grande parte de sua resistência.

\section{CONCLUSÕES}

Após os ensaios, conclui-se que o impacto das altas temperaturas na resistência à compressão do concreto foi desprezável na região da armadura e fios protendidos, camadas que chegaram a temperaturas inferiores a 100 C. Já nas camadas de 20 e $10 \mathrm{~mm}$, houve uma redução de resistência à compressão de, respectivamente, $25 \mathrm{e}$ $70 \%$, visto que as temperaturas chegaram a $700 \mathrm{C}$. Portanto, verificou-se que a estrutura continuava satisfazendo as condições de segurança, sendo necessária a reconstituição das camadas mais superficiais, a fim de manter a durabilidade da estrutura pós-sinistro.

Após a realização deste estudo, reforçou-se a hipótese de que ensaios de caracterização avançada apresentam indícios que podem ser relacionados com o efeito do fogo e de elevadas temperaturas em estruturas de concreto armado e protendido. Inicialmente, através da inspeção visual foi possível avaliar as principais características da edificação, da qual se identificou a ocorrência de desplacamento explosivo e a área de abrangência do sinistro. A complementação dos resultados por meio da caracterização química da matéria mostra-se, neste caso, precisa e importante para a confiabilidade dos valores encontrados. 
EHRENBRING, H. Z.; ORTOLAN, V.; BOLINA, F.; PACHECO, F.; GIL, A. M.; TUTIKIAN, B. F. revista Matéria, v.22, n.3, 2017.

\section{BIBLIOGRAFIA}

[1] MEHTA, P. K.., MONTEIRO, P. J., Concreto: microestrutura, propriedades e materiais, 1. ed., São Paulo, Pini, 2014.

[2] CALLISTER Jr., W. D., RETHWISCH, D. G., Fundamentos da ciência e engenharia de materiais: uma abordagem integrada, 1 ed.,. Rio de Janeiro, LTC, 2012.

[3] MORALES, G., CAMPOS, A., FAGANELlO, A. M. P., “A ação do fogo sobre os componentes de concreto”, Ciências exatas e Tecnológicas, v. 32, n. 1, pp. 47-55, Jan. 2011.

[4] BAMONTE, P., MONTE, F., "Lo. Reinforced concrete columns exposed to standard fire: Comparison among different constitutive models for concrete at high temperature", Fire Safety Journal, v. 71, n. 1, pp. 310-323, Abr. 2015.

[5] CASONATO, C. A., Ação de elevadas temperaturas em modelos de paredes de concreto e de alvenaria sob cargas de serviço, Dissertação de M.Sc., PPGEE/UFRGS, Porto Alegre, RS, Brasil, 2007.

[6] BOLINA, F. L., PRAGER, G. L., RODRIGUES, E., et al., "Evaluation off ire resistance of massive reinforced concrete walls", Ambiente Construído, v. 15, n. 4, pp. 291-305, Dez. 2015.

[7] BIOLZI, L., CATTANEO, S., ROSATI, G., "Evaluating residual properties of thermally damaged concrete", Cement and Concrete Composites, v. 30, n. 10, pp. 907-916, Nov. 2008.

[8] HAGER, I., "Behaviour of cement concrete at high temperature", Bulletin of the Polish Academy of Sciences: Technical Sciences, v. 61, n. 1, pp. 145-154, Mar. 2013.

[9] KIRCHHOF, L. D., Estudo teórico-experimental da influência do teor de umidade no fenômeno de spalling explosivo em concretos expostos a elevadas temperaturas, Tese de D.Sc., PPGEE/UFRGS, Porto Alegre, RS, Brasil, 2010.

[10] JANSSON, R., Fire spalling of concrete : theoretical and experimental studies, Tese de D.Sc., KTH Royal/ITS, Estocolmo, Suécia, 2013.

[11] KLINGSCH, E. W. H., Explosive spalling of concrete in fire, Tese de D.Sc., ETH ZURICH, Zurique, Alemanha, 2014.

[12] NINCE, A. A., Lascamento do concreto exposto a altas temperaturas, Tese de D.Sc., EPUSP/USP, São Paulo, SP, Brasil, 2006.

[13] GUO, Z., SHI, X., Experiment and calculation of reinforced concrete at elevated temperatures, 1 ed., Elsevier, Waltham, 2011.

[14] BOLINA, F. L., Avaliação experimental da influência dos requisitos de durabilidade na segurança contra incêndio de protótipos de pilares pré-fabricados de concreto armado, Dissertação de M.Sc., PPGAU/UNISINOS, São Leopoldo, RS, Brasil, 2016.

[15] FELICETTI, R., LO MONTE, F., "Concrete spalling: Interaction between tensile behaviour and pore pressure during heating", Concrete Spalling due to Fire Expositure, In: Anais $3^{\circ}$ International Workshop, 2013.

[16] FIB Bulletin 38: Fire design of concrete structures - materials, structures and modeling, FÉDÉRATION INTERNATIONALE DU BÉTON, Lausanne, 2007.

[17] LIM, S., MONDAL, P., "Micro-and nano-scale characterization to study the thermal degradation of cement-based materials", Materials Characterization, v. 92, n. 1, pp. 15-25, Feb. 2014.

[18] ZHANG, Q., YE, G., KOENDERS, E., "Investigation of the structure of heated Portland cement paste by using various techniques", Construction and Building Materials, v. 38, n. 2, pp. 1040-1050, Jun. 2013.

[19] MINAMI, K., TAZAWA, E., TERANISHI, S., "Effect of microscopic thermal stress on mechanical properties of concrete subjected to high temperature", Journal of Materials, Concrete Structures and Pavements, v. 13, n. 420, pp. 173-180, Aug. 1990.

[20] NEVILlE, A. M., BROOKS, J. J., Tecnologia do Concreto, 2 ed., Washington, Bookman, 2013.

[21] GRATTAN-BELLEW, P. E., "Microstructural investigation of deteriorated Portland cement concretes", Construction and Building Materials, v. 10, n. 1, pp. 3-16, Jan. 1996.

[22] ABNT, NBR 15200: Projeto de estruturas de concreto em situação de incêndio. Rio de Janeiro, 2012.

[23] EUROPEAN COMMITTEE FOR STANDARDIZATION (EN). BS EN 1992: Design of concrete structures - Part 1-2: General rules - Structural fire design: Eurocode 2. Bruxelas, British Standards Institution: 2004. 
[24] HANDOO, S. K., AGARWAL, S., AGARWAL, S. K., "Physicochemical, mineralogical, and morphological characteristics of concrete exposed to elevated temperatures", Cement and Concrete Research, v. 32, n. 7, pp. 1009-1018, Out. 2002.

[25] LIMA, R. C. A., Investigação do comportamento de concretos em temperaturas elevadas, Tese de D.Sc., PPGEE/UFRGS, Porto Alegre, RS, Brasil, 2005.

[26] SOUSA, M. M. de., Estudo experimental do comportamento mecânico e da microestrutura de um concreto convencional após simulação das condições de incêndio, Dissertação de M.Sc., PPGCC/UFMG, Belo Horizonte, MG, Brasil, 2009.

[27] ARIOZ, O., "Retained properties of concrete exposed to high temperatures: size effect", Fire and Materials, v. 33, n. 5, pp. 211-222, Aug. 2009.

[28] KIM, K. Y., YUN, T. S., PARK, K. P., "Evaluation of pore structures and cracking in cement paste exposed to elevated temperatures by X-ray computed tomography", Cement and Concrete Research, v. 50, n. 4, pp. 34-40, Jul. 2013.

[29] ANNEREL, E., TAERWE, L., "Revealing the temperature history in concrete after fire exposure by microscopic analysis", Cement and Concrete Research, v. 39, n. 12, pp. 1239-1249, Dez. 2009. 